\title{
Lung CCR6-CXCR3- Th2 Cells as an Indicator of Progressive Fibrosing Interstitial Lung Diseases: A Prospective Observational Study
}

\author{
Tsukie Kin Tsukuda \\ Kochi Medical School, Kochi University \\ Minoru Fujimoto \\ School of Medicine, Iwate Medical University \\ Hiroshi Ohnishi ( $\nabla$ honi@kochi-u.ac.jp ) \\ Kochi Medical School, Kochi University \\ Yu Nakatani \\ Kochi Medical School, Kochi University \\ Kazufumi Takamatsu \\ Kochi Medical School, Kochi University

\section{Tetsuji Naka} \\ School of Medicine, Iwate Medical University \\ Akihito Yokoyama \\ Kochi Medical School, Kochi University
}

\section{Research Article}

Keywords: bronchoalveolar lavage, forced vital capacity, progressive fibrosing interstitial lung disease, helper $\mathrm{T}$ type 2 cell

Posted Date: January 21st, 2022

DOI: https://doi.org/10.21203/rs.3.rs-1271280/v1

License: (9) This work is licensed under a Creative Commons Attribution 4.0 International License. Read Full License 


\section{Abstract}

\section{Background:}

Progressive fibrosing interstitial lung diseases have a poor prognosis and may be resistant to corticosteroids and/or immunosuppressants, but antifibrotic therapies such as nintedanib and pirfenidone have been shown to slow the deterioration of lung function. The aim of this study is to identify the characteristic cellular profile of bronchoalveolar lavage fluid at diagnosis for predicting progressive fibrosing interstitial lung diseases.

\section{Methods:}

Patients with interstitial lung diseases were divided into fibrotic and non-fibrotic phenotypes by chest high-resolution computed tomography. The progressive fibrosing phenotype was determined to be fibrotic diseases with more than a $5 \%$ relative decline in the percent predicted value of forced vital capacity over 6 months. The proportions of T cell, B cell, monocyte, and macrophage subsets and the degree of $\mathrm{T}$ cell activation in peripheral blood and bronchoalveolar lavage fluid were measured by flowcytometry.

\section{Results:}

Fibrotic interstitial lung diseases $(n=27)$ have a Th2-dominant phenotype, whereas non-fibrotic diseases $(n=13)$ have a Th1/Th17-dominant phenotype in bronchoalveolar lavage fluid. In patients with fibrotic diseases, the proportion of Th2 cells, but not the serum KL-6 level or the proportion of lymphocytes, was significantly higher in patients with progressive fibrosing phenotype than in those with non-progressive phenotype, and the receiver operating characteristic curve analysis showed an AUC of 0.79 . Thus, a greater population of Th2 cells in bronchoalveolar lavage fluid was diagnostic for progressive fibrosing phenotype.

\section{Conclusions:}

An increased proportion of Th2 cells in bronchoalveolar lavage fluid is associated with greater deterioration of lung function in fibrosing interstitial lung diseases.

\section{Trial registration:}

This study was was registered in the UMIN Clinical Trials Registry (Registry ID UMIN000041871) on October 1, 2020.

\section{Introduction}

Interstitial lung diseases (ILDs) consist of more than 200 lung diseases, most of which are associated with pulmonary fibrosis. Idiopathic pulmonary fibrosis (IPF) is classified as an idiopathic interstitial pneumonia (IIP) with the most frequent progressive fibrosing phenotype and the worst prognosis. Other 
ILDs such as idiopathic non-specific interstitial pneumonia (NSIP), connective tissue disease-associated ILD (CTD-ILD), unclassifiable IIPs, sarcoidosis, fibrotic hypersensitivity pneumonitis (HP), and pneumoconiosis often show similar fibrotic clinical features: progression of pulmonary fibrosis, worsening dyspnea on exertion, progressive decline in lung function, resistance to corticosteroids and/or immunosuppressive therapies, and poor prognosis (1-4). They are categorized as progressive fibrosing interstitial lung disease (PF-ILD) or ILDs with a progressive fibrosing phenotype, and antifibrotic therapies such as nintedanib and pirfenidone have been shown to improve disease progression of PF-ILD (5-7).

Prediction of future deterioration of lung function and/or progressive pulmonary fibrosis is difficult in real-world clinical settings, especially in patients with non-usual interstitial pneumonia (UIP) pattern on chest high-resolution computed tomography (HRCT), even though imaging resolution has improved remarkably. In such patients, sequential changes in pulmonary fibrosis on chest HRCT and/or in forced vital capacity (FVC) are often evaluated to determine whether antifibrotic therapy should be started, but this follow-up may delay the treatment of PF-ILD. Identifying PF-ILD at diagnosis is extremely important for improving patients' prognosis.

There is an urgent and unmet need to identify predictors of responsiveness to corticosteroids, immunosuppressants, and/or antifibrotic drugs. The underlying pathological mechanism of fibrosis in PF-ILD remains unclear and can be complicated by the involvement of multiple factors. Bronchoalveolar lavage fluid (BALF) containing various immune cells recovered from the lungs is the best research sample to identify such a mechanism. In the present study, cellular profiles of T cell, B cell, macrophage, and monocyte subsets were compared in BALF and the peripheral blood of patients with fibrotic and nonfibrotic ILDs using multicolor flow cytometry, and a characteristic cellular profile of helper $\mathrm{T}$ (Th) cells in BALF to predict PF-ILD was found.

\section{Methods}

\section{Patient population and collection of samples}

Patients with ILDs diagnosed in our hospital between 2018 and 2021 were enrolled in this study [ $\mathrm{n}=40$, IPF $(n=6), \operatorname{HP}(n=12)$, CTD-ILD $(n=14)$, sarcoidosis $(n=7)$, and pleuroparenchymal fibroelastosis (PPFE, $n=1)$ ). Diagnoses of IPF and HP were based on the Official ATS/ERS/JRS/ALAT Clinical Practice Guideline and the Official ATS/JRS/ALAT Clinical Practice Guideline, respectively $(8,9)$. Diagnosis of sarcoidosis was based on accepted criteria (10), and only cases with interstitial shadows in the lung fields were enrolled. Diagnosis of CTD-ILD was based on the common diagnostic criteria for each respective CTD and the presence of ILD on chest HRCT (11-15).

Participants underwent chest HRCT before bronchoscopy. BALF $(n=40)$ and peripheral blood $(n=38)$ were obtained from these patients on the same day or within 1 day. Serum Krebs von den Lungen-6 (KL-6) levels were measured by the chemiluminescent enzyme immunoassay LUMIPULSE KL- 6 using a KL-6 antibody (SEKISUI MEDICAL Co., Ltd, Tokyo, Japan). The cut-off value for serum KL- 6 was set at 435 $\mathrm{U} / \mathrm{ml}$ according to the manufacturer's instructions. Patients with coexisting active respiratory infections, 
patients being treated with antifibrotic drugs, and/or anticancer agents including immune checkpoint inhibitors, or insufficient BALF recovery (recovery rate less than $35 \%$ ) were excluded. Progressive fibrosing interstitial lung disease (PF-ILD) was defined as fibrotic ILD (traction bronchiectasis, architectural distortion, and/or honeycombing) on chest HRCT and a relative percent predicted value of forced vital capacity (\%FVC) decrease of $5 \%$ or more over 6 months despite disease management deemed appropriate by the physician (e.g., antigen avoidance and treatment with corticosteroids \pm immunosuppressive agents) in this study.

This study was approved by the ethics committee of the Kochi Medical School, Kochi University (Approval number 202-102) and was registered in the UMIN Clinical Trials Registry (Registry ID UMIN000041871). Written, informed consent was obtained from all participants.

\section{Interpretation of chest HRCT images}

Chest HRCT findings were reviewed separately by two independent respiratory specialists who were not aware of the patients' profiles and grouped into fibrotic and non-fibrotic ILDs. The presence of traction bronchiectasis, architectural distortion, and/or honeycombing on chest HRCT provided evidence of lung fibrosis based on the guideline of the Fleischner Society. Cases with traction bronchiectasis and/or architectural distortion without honeycombing were further defined as fibrotic NSIP (f-NSIP) patterns, whereas cases with honeycombing were defined as a UIP pattern. Following the initial independent chest HRCT assessment, discordant evaluations were resolved by consensus reached after consultation between the two observers.

\section{Flow cytometry}

For staining of cell surface markers, cells in BALF and whole blood were incubated with the following fluorescent-conjugated anti-human antibodies (CD3-FITC, clone UCHT1; CD45-PerCP-Cy5.5, clone HI30; CD56-PE, clone HCD56; HLA-DR-PE-Dazzle594, clone L243; CD4-PE-Cy7,clone SK3; CD197-AF647, clone G043H7; CD45RA-BV421, clone HI100; CD8-BV510, clone RPA-T8; CD38-BV60, clone HIT2; CD38-FITC, clone HIT2; CD24-PerCP/Cy5, clone ML5; IgD-PE, clone IA6-2; CD20-PE-Cy7, clone 2H7; CD138-APC, clone MI15; CD27-APC-Cy7, clone 0323; CD19-BV421, clone HIB19; CD25-FITC, clone BC96; CD185-PerCP-Cy5.5, clone J252D4; CD127-PE-Cy7, clone A019D5; CD3-APC-Cy7, clone UCHT1; CD4-BV510, clone OKT4; CD25BV605, cloneBC96; CD19-FITC, clone HIB19; CD56-FITC, clone HCD56; CD66a/c/e-Alexa Fluor488, clone ASL-32; CD11 c-PE, clone 3.9; CD169-PE, clone 7-239; CD14-APC, clone HCD14; HLA-DR-APC-Cy7, clone L243; CD206-BV421, clone 15-2; CD16-BV510, clone 3G8; CD123-BV421, clone 6H6 [BioLegend, San Diego, CA]; CD196-PE, clone11A9; CD183-APC, clonelC6/CXCR3; CCR4-BV421, clone1G1 [BD Pharmingen, San Jose, CA]). See the online supplement for details of the procedure. The FACSAria Fusion and FACS LSRFortessa (BD Biosciences, San Jose, CA) and FlowJo software (Tree Star, Inc., Ashland, OR) were used for flow cytometric analyses. The gating strategy for identifying Th subsets is shown in Figure 1 [peripheral blood (A) and BALF (B)], and that for the other staining sets is shown in Figure E1 (T cell subsets classified by maturity stage) and E2 (A: B cell subtypes, B: monocyte and dendritic cell (DC) subpopulations, C: alveolar macrophage in BALF). Details of the gating steps for identifying Th subsets 
are shown in Figure 1. Classification of Th subsets (Th1, Th1/17, Th17, and Th2) of conventional T cells by CCR6 and CXCR3 expressions is shown in Table 1(16).

\section{Pulmonary function tests}

Pulmonary function tests were performed 6-7 months before bronchoscopy as a baseline and a second time around 1 month before bronchoscopy in 11 cases, and around 1 month after bronchoscopy as a baseline and a second time 6-7 months after bronchoscopy in 10 cases.

\section{Statistical analysis}

Data are presented as medians (interquartile range). Differences between two groups were evaluated by the Mann-Whitney $U$ test. The correlation coefficients between blood cells and BALF cells were calculated using Spearman's rank correlation coefficient (Rs). Quantitative differences were tested by the Chisquared test for goodness of fit or by Fisher's exact test. Interobserver agreement for chest HRCT findings was assessed using Kappa statistics. Kappa values greater than 0.61 were considered to indicate good agreement between observers. Receiver operating characteristic (ROC) curve analysis was used to determine the cut-off values that provide the optimal diagnostic accuracy for the proportion of Th2 cells in BALF to predict PF-ILD. All statistical analyses were performed using EZR version 1.54 (Saitama Medical Center, Jichi Medical University, Saitama, Japan) (17). Statistical significance was set at $p<$ 0.05 .

\section{Results}

\section{Patients' characteristics}

Chest HRCT findings were classified as fibrotic ILD in 27 patients and non-fibrotic ILD in 13 patients (Table 2). Interobserver variability before reaching consensus agreement, expressed as a Kappa value, was 0.74 , indicating good agreement between observers. There was no significant difference in age between fibrotic ILD and non-fibrotic ILD. There were more males in fibrotic ILD than in non-fibrotic ILD, and more never smokers in non-fibrotic ILD than in fibrotic ILD. Serum KL-6 and lactate dehydrogenase (LDH) were significantly higher in fibrotic ILD than in non-fibrotic ILD. In cellular profiles of BALF, the proportion of lymphocytes was significantly higher in non-fibrotic ILD than in fibrotic-ILD, whereas the proportion of eosinophils and neutrophils was significantly higher in fibrotic ILD than in non-fibrotic-ILD. IPF, HP, and CTD-ILD were common underlying diseases of fibrotic ILD, whereas sarcoidosis was the most common underlying disease of non-fibrotic ILD.

Furthermore, relative decline of \% forced vital capacity over 6 months, chest HRCT pattern, cellular profile of bronchoalveolar lavage fluid, and serum KL-6 levels in fibrotic patients with fibrotic ILDs are shown in Table $3(n=21)$. At the time of bronchoscopy, only one of the patients with fibrotic ILD was treated with corticosteroids and tacrolimus; the others were untreated. After bronchoscopy, some were started on corticosteroids alone or in combination with corticosteroids and tacrolimus. 


\section{Th subsets in BALF and fibrotic phenotype on chest HRCT}

Cellular profiles of BALF showed differences in the proportion of Th subsets between fibrotic ILD and non-fibrotic ILD on chest HRCT. The proportion of CCR6 ${ }^{-}$CXCR3 ${ }^{-}$CD $4^{+} T$ cells (Th2) was significantly increased in BALF of fibrotic ILD compared with non-fibrotic ILD (Figure 2A). On the other hand, the proportion of $\mathrm{CCR}^{+} \mathrm{CXCR}^{+} \mathrm{CD} 4^{+} \mathrm{T}$ cells (Th1/Th17) was significantly increased in BALF of non-fibrotic ILD compared with fibrotic ILD (Figure 2B). These differences in Th subsets were not observed in cells in peripheral blood when comparing fibrotic and non-fibrotic ILD (Figure 2A and 2B). The proportion of Th1/Th17 $\left(\right.$ CCR6 $^{+} \mathrm{CXCR}^{+}{ }^{\mathrm{T}}$ cells) in BALF, but not of Th2 (CCR6 ${ }^{-\mathrm{CXCR}^{-}}{ }^{\mathrm{T}}$ cells, Rs $=0.181, p=0.276$ for Th2, Figure 2C), was weakly correlated with the proportion in peripheral blood (Rs $=0.485, p=0.0021$ for Th1/Th17, Figure 2D). Cellular profiles in subsets of B cells, macrophages, or monocytes in the peripheral blood and BALF were not different between patients with fibrotic ILD and non-fibrotic ILD. These results suggest that the Th2 subset in the lung, but not in peripheral blood, is associated with the fibrotic phenotype of ILD on chest HRCT.

\section{Proportion of lymphocytes and Th subsets in BALF in all ILD patients}

Furthermore, since the proportion of lymphocytes in BALF is often used as a criterion to predict response to corticosteroids in clinical practice, the correlations between the proportion of lymphocytes and Th2 and Th1/17 in BALF were examined. The proportion of Th2 (CCR6 ${ }^{-} \mathrm{CXCR} 3^{-} \mathrm{CD}^{+}{ }^{+} \mathrm{T}$ cells) was negatively correlated with the proportion of lymphocytes in BALF in all study subjects $(\mathrm{n}=40, \mathrm{Rs}=-0.419, p=0.0071$, Figure 3A), but no correlation was found for Th1/Th17 $\left(\mathrm{CCR6}^{+} \mathrm{CXCR} 3^{+} \mathrm{CD} 4^{+} \mathrm{T}\right.$ cells $)(\mathrm{Rs}=0.211, p=$ 0.191 , Figure $3 B$ ). These results suggest that the lower the lymphocytosis, the higher the Th2 in BALF in patients with ILDs.

\section{Th subsets in BALF and PF-ILD phenotype in patients with fibrotic ILD}

Based on these results, we hypothesized that Th2 (CCR6 ${ }^{-} \mathrm{CXCR} 3^{-} \mathrm{CD} 4^{+} \mathrm{T}$ cells) in BALF could be an indicator of PF-ILD and then verified this hypothesis.

In fibrotic ILD patients, the proportion of Th2 (CCR6 ${ }^{-} \mathrm{CXCR}^{-} \mathrm{T}$ cells) in BALF was significantly higher in patients with PF-ILD (a relative \%FVC decline of $5 \%$ or more over 6 months) than in patients with non PFILD (less reduction) ( $p=0.028$, Figure $4 A$ ). The proportion of lymphocytes in BALF and serum KL-6 levels were not different between patients with PF-ILD and non PF-ILD (Figure 4B and C). These results suggest that the Th2 subset in the lungs, but not lymphocytes in BALF or the serum KL-6 level, may be associated with the PF-ILD phenotype in fibrotic ILD.

\section{Diagnostic value of Th2 cells in BALF for determining PF-ILD}

ROC curve analysis was used to evaluate the diagnostic value of the proportion of Th2 cells in BALF for identifying PF-ILD patients, and the area under the curve (AUC) value calculated from ROC curve analysis was 0.787 [95\% confidence interval (CI): 0.565-1.000] (Figure 4D). The cut-off value of the proportion of 
Th2 cells in BALF was set at more than 14.80 based on the highest Youden's index; the sensitivity, specificity, and likelihood ratio for predicting PF-ILD were $0.889,0.750$, and 3.56 , respectively. Even with the small number of patients included in this study, these data suggest that Th2 in BALF has moderate diagnostic accuracy for PF-ILD.

\section{Discussion}

The cellular profiles of BALF and peripheral blood were evaluated in mostly treatment-naïve patients with various ILDs, and it was found that the proportion of Th2 (CCR6 ${ }^{-}$CXCR3 ${ }^{-} C D 4^{+} T$ ) cells in BALF, not of other Th subsets, was significantly increased in patients with fibrotic ILD compared with non-fibrotic ILD on chest HRCT. and they were increased with a significant decrease in \%FVC. These results clearly demonstrated that the predominance of Th2 cells in the lungs was associated with progressive lung fibrosis in patients with ILD. The increased proportion of CCR6 ${ }^{-}$CXCR3-Th2 cells in BALF found in this study may help to identify these patients and provide early intervention to improve the prognosis of PFILD patients, but further confirmation is needed in the future.

In general, lymphocytosis in BALF is an important predictor for responsiveness to corticosteroids in clinical practice, but the present study demonstrated that the proportion of Th2 in BALF, but not of lymphocytes, may be predictive of progressive fibrosing phenotype. Although serial measurements of serum KL-6 levels were reported to be useful for predicting progression of pulmonary fibrosis $(18,19)$, serum KL-6 levels taken at the time of bronchoscopy in this study did not show an association with the progression of pulmonary fibrosis.

PF-ILD was found to have both fibrotic ILD (traction bronchiectasis, architectural distortion, and/or honeycombing) on chest HRCT and a relative \%FVC decrease of $5 \%$ or more over 6 months despite disease management deemed appropriate by the physician (e.g., antigen avoidance and treatment with corticosteroids \pm immunosuppressive agents) in the present study, but there are no standard criteria for PF-ILD. One paper has proposed any of the following criteria within 24 months as evidence of disease progression: a relative decline of $10 \%$ or greater in FVC; a relative decline of $15 \%$ or greater in diffusing capacity of the lung for carbon monoxide; worsening symptoms; or radiological appearance accompanied by a $5 \%$ or greater but less than $10 \%$ relative decrease in FVC (4). However, another study of pirfenidone used a criterion of more than a 5\% FVC decrease for at least 6 months (20).

In the present study, the proportion of Th2 among conventional T cells in BALF was increased with the progression of fibrosis. Interestingly, several reports have demonstrated the relationship between pulmonary fibrosis and Th2. Periostin, induced by the type 2 cytokines interleukin-4 (IL-4) and IL-13, has been reported to be involved in pulmonary fibrosis with crosstalk of TGF- $\beta(21,22)$, and serum periostin could be a good predictor of decreased lung function, increased radiation fibrosis, and prognosis (23-26). Moreover, lines of evidence indicate that M2-like macrophages, known to be induced by type 2 cytokines, are profoundly involved in the pathogenesis of fibrosis (27-29). At present, which cell type(s) drive the pathogenesis of lung fibrosis remains unknown. In the present study, the absolute number of BALF Th2 
was not markedly increased in patients with progressive fibrosis, given that the proportion of BALF lymphocytes in these patients was lower than of other ILD patients. Therefore, although a relative increase in BALF Th2 is likely an indicator of the Th2-dominant pathology in progressive fibrosis, Th2type $T$ cells may not be the main player in the progression of the disease. Further studies are needed to define the pathological roles of Th2-type T cells in lung fibrosis.

Currently, antifibrotic agents such as pirfenidone and nintedanib have been reported to be effective in reducing lung function decline in PF-ILD. The timing of the introduction of antifibrotic agents in clinical practice depends on the individual judgment of the attending physicians, and many believe that it is best to start as early as possible if the disease has progressive fibrosing features. Since poorer performance status is the most common cause of the discontinuation of treatment, it is important to start antifibrotic drugs early before performance status worsens (30).

The limitations of this study include the small number of study subjects in a single center dealing with respiratory diseases. Even though a small number of subjects were included, the present study clearly showed that Th2 cells in BALF had moderate diagnostic accuracy for determining PF-ILD. The second limitation is that the effect of antifibrotic drugs on Th2-high ILD patients could not be investigated in the present study, because only one patient was treated with nintedanib. Another limitation is the heterogeneity of underlying ILD. Different backgrounds of the diseases may have their own specific immunological responses, which may have affected the study results. Therefore, further large prospective studies are required to examine the administration of antifibrotic drugs to ILD patients with high Th2 cells in BALF.

\section{Conclusion}

In conclusion, the predominance of Th2 cells in BALF is a predictor of a greater decrease of lung function in PF-ILD, and these patients can be good candidates for antifibrotic therapy.

\section{Abbreviations}

AUC, area under the curve; BALF, bronchoalveolar lavage fluid; BF, bronchoscopy; CTD-ILD, connective tissue disease-associated ILD; f-NSIP, fibrotic NSIP; FVC, forced vital capacity; Th, helper T; HRCT, highresolution computed tomography; HP, hypersensitivity pneumonitis; IIP, idiopathic interstitial pneumonia; IPF, idiopathic pulmonary fibrosis; IL, interleukin; ILD, interstitial lung disease; KL-6, Krebs von den Lungen6; LDH, lactate dehydrogenase; NSIP, non-specific interstitial pneumonia; PPFE, pleuroparenchymal fibroelastosis; PF-ILD, progressive fibrosing interstitial lung disease; ROC, Receiver operating characteristic; UIP, usual interstitial pneumonia.

\section{Declarations}

\section{Ethics approval and consent to participate}


This study was approved by the ethics committee of the Kochi Medical School, Kochi University. Written informed consent was obtained from all participants.

\section{Consent for publication}

Not applicable

\section{Availability of data and materials}

The datasets used and/or analyzed during the current study are available from the corresponding author on reasonable request.

\section{Competing interests}

The authors declare that they have no competing interests.

\section{Funding}

This work was supported by Kochi University Hospital and Research funds from the Ministry of Education, Culture, Sports, Science and Technology of Japan

\section{Author Contributions}

Study concept and design: TKT and MF; recruitment of subjects: TKT, HO, YN, and KT; acquisition and analysis of flowcytometric data: TKT and MF; acquisition of clinical data: TKT, HO, YN, KT, and AY; analysis and interpretation of data: TKT and MF; first drafting of the manuscript: TKT, HO and MF; critical revision of the manuscript for important intellectual content: all authors; obtained funding: TN and AY; study supervision: HO, MF, TN, and AY; data access and responsibility: TKT and HO had full access to all the data in the study and take responsibility for the integrity of the data and the accuracy of the data analysis.

\section{Acknowledgments}

The authors would like to thank all patients who enrolled in this study. They also thank Prof. Yoshiyasu Okuhara, Yuki Hyodoh, Yumi Komatsu, Ayumi Quick, Motoki Kondo, and the medical staff of the Department of Respiratory Medicine and Allergology, Kochi Medical School for their help.

\section{References}

1. Walsh SL, Sverzellati N, Devaraj A, Keir GJ, Wells AU, Hansell DM. Connective tissue disease related fibrotic lung disease: high resolution computed tomographic and pulmonary function indices as prognostic determinants. Thorax. 2014;69:216-2.

2. Travis WD, Costabel U, Hansell DM, King TE Jr, Lynch DA, Nicholson AG, et al. An official American Thoracic Society/European Respiratory Society statement: Update of the international 
multidisciplinary classification of the idiopathic interstitial pneumonias. Am J Respir Crit Care Med. 2013;188:733-48.

3. Kim EJ, Elicker BM, Maldonado F, Webb WR, Ryu JH, Van Uden JH, et al. Usual interstitial pneumonia in rheumatoid arthritis-associated interstitial lung disease. Eur Respir J. 2010;35:1322-8.

4. Flaherty KR, Brown KK, Wells AU, Clerisme-Beaty E, Collard HR, Cottin V, et al. Design of the PF-ILD trial: a double-blind, randomised, placebo-controlled phase III trial of nintedanib in patients with progressive fibrosing interstitial lung disease. BMJ Open Respir Res. 2017;4:e000212.

5. Flaherty KR, Wells AU, Cottin V, Devaraj A, Walsh SLF, Inoue Y, et al. Nintedanib in Progressive Fibrosing Interstitial Lung Diseases. N Engl J Med. 2019;381:1718-27.

6. Neighbors M, Cabanski CR, Ramalingam TR, Sheng XR, Tew GW, Gu C, et al. Prognostic and predictive biomarkers for patients with idiopathic pulmonary fibrosis treated with pirfenidone: posthoc assessment of the CAPACITY and ASCEND trials. Lancet Respir Med. 2018;6:615-26.

7. Idiopathic Pulmonary Fibrosis Clinical Research Network, Raghu G, Anstrom KJ, King TE Jr, Lasky JA, Martinez FJ. Prednisone, azathioprine, and N-acetylcysteine for pulmonary fibrosis. N Engl J Med. 2012;366:1968-77.

8. Raghu G, Remy-Jardin M, Myers JL, Richeldi L, Ryerson CJ, Lederer DJ, et al. Diagnosis of Idiopathic Pulmonary Fibrosis. An Official ATS/ERS/JRS/ALAT Clinical Practice Guideline. Am J Respir Crit Care Med. 2018;198:e44-e68.

9. Raghu G, Remy-Jardin M, Ryerson CJ, Myers JL, Kreuter M, Vasakova M, et al. Diagnosis of Hypersensitivity Pneumonitis in Adults. An Official ATS/JRS/ALAT Clinical Practice Guideline. Am J Respir Crit Care Med. 2020;202:e36-e69.

10. Statement on sarcoidosis. Joint Statement of the American Thoracic Society (ATS), the European Respiratory Society (ERS) and the World Association of Sarcoidosis and Other Granulomatous Disorders (WASOG) adopted by the ATS Board of Directors and by the ERS Executive Committee, February 1999. Am J Respir Crit Care Med. 1999;160:736-55.

11. Arnett FC, Edworthy SM, Bloch DA, McShane DJ, Fries JF, Cooper NS, et al. The American Rheumatism Association 1987 revised criteria for the classification of rheumatoid arthritis. Arthritis Rheum. 1988;31:315-24.

12. Bohan A, Peter JB. Polymyositis and dermatomyositis (first of two parts). N Engl J Med. 1975;292:344-7.

13. Smolen JS, Steiner G. Mixed connective tissue disease: to be or not to be? Arthritis Rheum. 1998;41:768-77.

14. Vitali C, Bombardieri S, Jonsson R, Moutsopoulos HM, Alexander EL, Carsons SE, et al. Classification criteria for Sjogren's syndrome: a revised version of the European criteria proposed by the AmericanEuropean Consensus Group. Ann Rheum Dis. 2002;61:554-8.

15. van den Hoogen F, Khanna D, Fransen J, Johnson SR, Baron M, Tyndall A, et al. 2013 classification criteria for systemic sclerosis: an American College of Rheumatology/European League against Rheumatism collaborative initiative. Arthritis Rheum. 2013;65:2737-47. 
16. 16. Maecker HT, McCoy JP, Nussenblatt R. Standardizing immunophenotyping for the Human Immunology Project. Nat Rev Immunol. 2012;12:191-200.

17. Kanda Y. Investigation of the freely available easy-to-use software 'EZR' for medical statistics. Bone Marrow Transplant. 2013;48:452-8.

18. Kuwana M, Shirai Y, Takeuchi T. Elevated Serum Krebs von den Lungen-6 in Early Disease Predicts Subsequent Deterioration of Pulmonary Function in Patients with Systemic Sclerosis and Interstitial Lung Disease. J Rheumatol. 2016;43:1825-31.

19. Yokoyama A, Kohno N, Hamada H, Sakatani M, Ueda E, Kondo K, et al. Circulating KL-6 predicts the outcome of rapidly progressive idiopathic pulmonary fibrosis. Am J Respir Crit Care Med. 1998;158:1680-4.

20. Maher TM, Corte TJ, Fischer A, Kreuter M, Lederer DJ, Molina-Molina M, et al. Pirfenidone in patients with unclassifiable progressive fibrosing interstitial lung disease: design of a double-blind, randomised, placebo-controlled phase II trial. BMJ Open Respir Res. 2018;5:e000289.

21. Takayama G, Arima K, Kanaji T, Toda S, Tanaka H, Shoji S, et al. Periostin: a novel component of subepithelial fibrosis of bronchial asthma downstream of IL-4 and IL-13 signals. J Allergy Clin Immunol. 2006;118:98-104.

22. Yoshihara T, Nanri Y, Nunomura S, Yamaguchi Y, Feghali-Bostwick C, Ajito K, et al. Periostin plays a critical role in the cell cycle in lung fibroblasts. Respir Res. 2020;21:38.

23. Ohta S, Okamoto M, Fujimoto K, Sakamoto N, Takahashi K, Yamamoto H, et al. The usefulness of monomeric periostin as a biomarker for idiopathic pulmonary fibrosis. PLoS One. 2017;12:e0174547.

24. Okamoto M, Hoshino T, Kitasato Y, Sakazaki Y, Kawayama T, Fujimoto K, et al. Periostin, a matrix protein, is a novel biomarker for idiopathic interstitial pneumonias. Eur Respir J. 2011;37:1119-27.

25. Okamoto M, Izuhara K, Ohta S, Ono J, Hoshino T. Ability of Periostin as a New Biomarker of Idiopathic Pulmonary Fibrosis. Adv Exp Med Biol. 2019;1132:79-87.

26. Tajiri M, Okamoto M, Fujimoto K, Johkoh T, Ono J, Tominaga M, et al. Serum level of periostin can predict long-term outcome of idiopathic pulmonary fibrosis. Respir Investig. 2015;53:73-81.

27. Misharin AV, Morales-Nebreda L, Reyfman PA, Cuda CM, Walter JM, McQuattie-Pimentel AC, et al. Monocyte-derived alveolar macrophages drive lung fibrosis and persist in the lung over the life span. J Exp Med. 2017;214:2387-404.

28. Osterholzer JJ, Olszewski MA, Murdock BJ, Chen GH, Erb-Downward JR, Subbotina N, et al. Implicating exudate macrophages and Ly-6C(high) monocytes in CCR2-dependent lung fibrosis following gene-targeted alveolar injury. J Immunol. 2013;190:3447-57.

29. Meziani L, Mondini M, Petit B, Boissonnas A, Thomas de Montpreville V, Mercier O, et al. CSF1R inhibition prevents radiation pulmonary fibrosis by depletion of interstitial macrophages. Eur Respir J. 2018;51:1702120.

30. Oishi K, Hirano T, Murata Y, Hamada K, Uehara S, Suetake R, et al. Medication persistence rates and predictive factors for discontinuation of antifibrotic agents in patients with idiopathic pulmonary fibrosis: a real-world observational study. Ther Adv Respir Dis. 2019;13:1753466619872890. 


\section{Tables}

Table 1. Definition of helper T cell subsets based on the surface expression of chemokine receptors

\begin{tabular}{|lll|}
\hline & CCR6 & CXCR3 \\
\hline Th1 & - & + \\
\hline Th1/17 & + & + \\
\hline Th17 & + & - \\
\hline Th2 & - & - \\
\hline
\end{tabular}

Table 2. Patients' characteristics and cellular profiles of bronchoalveolar lavage fluid by chest highresolution computed tomography patterns

TCC, total cell counts; ILD, fibrotic interstitial lung diseases; c-NSIP, cellular nonspecific interstitial pneumonia; f-NSIP, fibrotic nonspecific interstitial pneumonia; UIP, usual interstitial pneumonia; IPF, idiopathic pulmonary fibrosis; HP, hypersensitivity pneumonitis; CTD, connective tissue disease; RA, rheumatoid arthritis; SSc, systemic sclerosis; PM/DM, polymyositis/dermatomyositis; SjS, Sjögren's syndrome; MCTD, mixed connective tissue disease; PPFE: Pleuroparenchymal fibroelastosis; BALF, bronchoalveolar lavage fluid.

${ }^{*} \mathrm{p}$-values are for comparisons between non-fibrotic ILD and fibrotic ILD.

Table 3. Relative decline of \% forced vital capacity over 6 months, chest high-resolution computed tomography pattern, cellular profile of bronchoalveolar lavage fluid, and serum KL-6 levels in patients with fibrotic interstitial lung diseases 


\begin{tabular}{|c|c|c|c|c|c|}
\hline \multirow{3}{*}{ Chest HRCT pattern } & \multicolumn{2}{|c|}{$\begin{array}{l}\text { Non-fibrotic ILD } \\
n=13\end{array}$} & \multicolumn{2}{|l|}{$\begin{array}{l}\text { Fibrotic ILD } \\
n=27\end{array}$} & \multirow{3}{*}{$\begin{array}{l}p \text { - } \\
\text { values* }\end{array}$} \\
\hline & c-NSIP & Others & f-NSIP & UIP & \\
\hline & $n=5$ & $n=8$ & $n=8$ & $n=19$ & \\
\hline Age (y) median (interquartile range) & $65(33)$ & $74(25)$ & $65.5(44)$ & $73(41)$ & 0.84 \\
\hline Male (\%) & $1(20)$ & $1(12.5)$ & $3(37.5)$ & $\begin{array}{l}14 \\
(73.7)\end{array}$ & 0.005 \\
\hline \multicolumn{6}{|l|}{ Smoking status } \\
\hline Never/Former/Current & $3 / 2 / 0$ & $5 / 3 / 1$ & $2 / 6 / 0$ & $2 / 16 / 1$ & 0.012 \\
\hline KL-6 [U/D] (median, range) & $\begin{array}{l}1111 \\
(10162)\end{array}$ & $\begin{array}{l}369 \\
(299)\end{array}$ & $\begin{array}{l}1592.5 \\
(13438)\end{array}$ & $\begin{array}{l}839 \\
(3931)\end{array}$ & 0.0037 \\
\hline LDH [U/l] (median, range) & $228(98)$ & $199(78)$ & $298(486 \rrbracket$ & $\begin{array}{l}238 \\
(140)\end{array}$ & 0.019 \\
\hline \multicolumn{6}{|l|}{$\begin{array}{l}\text { BALF cellular profile: median } \\
\text { (interquartile range) }\end{array}$} \\
\hline $\mathrm{TCC} / \times 10^{5} \mathrm{ml}$ & $\begin{array}{l}1.38 \\
(14.7)\end{array}$ & $\begin{array}{l}3.88 \\
(7.75)\end{array}$ & $2.54(5.39)$ & $\begin{array}{l}3.05 \\
(5.83)\end{array}$ & 0.73 \\
\hline Lymphocytes (\%) & $\begin{array}{l}38.8 \\
(56.5)\end{array}$ & $\begin{array}{l}47.0 \\
(87.9)\end{array}$ & $27.4(83.9)$ & $\begin{array}{l}13.5 \\
(72.2)\end{array}$ & 0.03 \\
\hline Macrophages (\%) & $\begin{array}{l}59.9 \\
(70.2)\end{array}$ & $\begin{array}{l}52.2 \\
(85.7)\end{array}$ & $60.8(80.4)$ & $\begin{array}{l}73.8 \\
(81.2)\end{array}$ & 0.57 \\
\hline Eosinophils (\%) & $0.3(9.7)$ & $0.3(1.5)$ & $1.55(20.3)$ & $2.3(14)$ & 0.003 \\
\hline Neutrophils (\%) & $1(10.5)$ & $0.9(5.5)$ & $2.65(31.5)$ & $\begin{array}{l}3.3 \\
(45.8)\end{array}$ & 0.003 \\
\hline \multicolumn{6}{|l|}{ Diagnosis of underlying disease } \\
\hline IPF & 0 & 0 & 0 & 6 & 0.62 \\
\hline $\mathrm{HP}$ & 2 & 1 & 2 & 8 & 0.41 \\
\hline \multicolumn{6}{|l|}{ CTD-ILD } \\
\hline SSc & 2 & 0 & 1 & 3 & 0.67 \\
\hline PM/DM & 0 & 0 & 3 & 0 & 0.62 \\
\hline SjS & 1 & 0 & 0 & 1 & 1 \\
\hline RA & 0 & 0 & 1 & 0 & 0.62 \\
\hline MCTD & 0 & 0 & 1 & 0 & 0.62 \\
\hline
\end{tabular}




\begin{tabular}{|llllll|} 
PPFE & 0 & 0 & 0 & 1 & 0.62 \\
\hline Sarcoidosis & 0 & 7 & 0 & 0 & 0.62 \\
\hline
\end{tabular}

Page $14 / 20$ 


\begin{tabular}{|c|c|c|c|c|c|c|}
\hline Diagnosis & $\begin{array}{l}\text { Chest } \\
\text { HRCT } \\
\text { pattern }\end{array}$ & $\begin{array}{l}\text { \%FVC decline } \\
(\%) \\
\text { / Time of PFT }\end{array}$ & Lymphocytes (\%) & $\begin{array}{l}\text { Th2 } \\
\text { (\%) }\end{array}$ & $\begin{array}{l}\text { Serum } \\
\text { KL-6 } \\
\text { level } \\
\text { (U/ml) }\end{array}$ & Treatment \\
\hline CTD-ILD & fNSIP & $\begin{array}{l}-41.6 \\
\text { / before and } 6 \\
\text { months after BF }\end{array}$ & 33.8 & 50.9 & 2476 & $\begin{array}{l}\text { PSL was started } \\
\text { after BF }\end{array}$ \\
\hline CTD-ILD & fNSIP & $\begin{array}{l}-24.9 \\
\text { / before and } 6 \\
\text { months after BF }\end{array}$ & 33.3 & 0.53 & 532 & $\begin{array}{l}\text { PSL and TAC were } \\
\text { started after BF }\end{array}$ \\
\hline CTD-ILD & fNSIP & $\begin{array}{l}-15.8 \\
\text { / before and } 6 \\
\text { months after BF }\end{array}$ & 8.8 & 6.52 & 843 & $\begin{array}{l}\text { PSL was started } \\
\text { after BF }\end{array}$ \\
\hline IPF & UIP & $\begin{array}{l}-7.03 \\
\text { / } 6 \text { months } \\
\text { before and the } \\
\text { day of } B F\end{array}$ & 13.5 & 9.02 & 2117 & None \\
\hline CTD-ILD & UIP & $\begin{array}{l}-3.9 \\
\text { / before and } 6 \\
\text { months after BF }\end{array}$ & 27.3 & 62.7 & 1534 & None \\
\hline IPF & UIP & $\begin{array}{l}-0.6 \\
/ 7 \text { months and } \\
1 \text { month before } \\
\mathrm{BF}\end{array}$ & 12.5 & 14.5 & 1354 & None \\
\hline HP & UIP & $\begin{array}{l}-0.03 \\
\text { / } 6 \text { months } \\
\text { before BF to the } \\
\text { day before }\end{array}$ & 23.8 & 9.53 & 4295 & Antigen avoidance \\
\hline IPF & UIP & $\begin{array}{l}-0.019 \\
\text { / before and } 6 \\
\text { months after BF }\end{array}$ & 13.5 & 13.4 & 839 & None \\
\hline HP & UIP & $\begin{array}{l}-0.2 \\
\text { / before and } 6 \\
\text { months after BF }\end{array}$ & 67.2 & 15 & 602 & Antigen avoidance \\
\hline HP & UIP & $\begin{array}{l}2.3 \\
\text { / before and } 6 \\
\text { months after BF }\end{array}$ & 9.3 & 7.91 & 1800 & Antigen avoidance \\
\hline CTD-ILD & fNSIP & 2.9 & 39.3 & 2.13 & 957 & None \\
\hline
\end{tabular}




\begin{tabular}{|c|c|c|c|c|c|c|}
\hline & & $\begin{array}{l}\text { / before and } 6 \\
\text { months after BF }\end{array}$ & & & & \\
\hline $\mathrm{HP}$ & UIP & 3.4 & 2.0 & 11.9 & 2635 & Antigen avoidance \\
\hline & & $\begin{array}{l}\text { / before and } 6 \\
\text { months after BF }\end{array}$ & & & & \\
\hline CTD-ILD & UIP & & 3 & 87.3 & 361 & PSL and TAC were \\
\hline & & $\begin{array}{l}\text { / } 7 \text { months and } \\
1 \text { month before } \\
\text { BF }\end{array}$ & & & & before BF \\
\hline $\mathrm{HP}$ & UIP & 9.2 & 74.2 & 44.4 & 921 & $\begin{array}{l}\text { PSL was started } \\
\text { after BF }\end{array}$ \\
\hline & & $\begin{array}{l}\text { / } 7 \text { months and } \\
1 \text { month before } \\
\text { BF }\end{array}$ & & & & \\
\hline $\mathrm{HP}$ & UIP & 9.6 & 33.0 & 3.61 & 534 & PSL was started \\
\hline & & $\begin{array}{l}/ 6 \text { months and a } \\
\text { week before BF }\end{array}$ & & & & \\
\hline CTD-ILD & fNSIP & 11.2 & 11 & 42 & 1385 & mPSL pulse and \\
\hline & & $\begin{array}{l}/ 6 \text { months and a } \\
\text { week before BF }\end{array}$ & & & & $\begin{array}{l}\text { and TAC was } \\
\text { started after BF }\end{array}$ \\
\hline $\mathrm{HP}$ & UIP & 12.8 & 16.8 & 73.9 & 1552 & Antigen avoidance \\
\hline & & $\begin{array}{l}\text { / } 7 \text { months and } \\
1 \text { month before } \\
\text { BF }\end{array}$ & & & & $\begin{array}{l}\text { PSL started after } \\
\text { exacerbation. }\end{array}$ \\
\hline IPF & UIP & 13 & 12.3 & 21.9 & 1250 & None \\
\hline & & $\begin{array}{l}\text { / } 6 \text { months } \\
\text { and a week } \\
\text { before BF }\end{array}$ & & & & \\
\hline HP & UIP & 13.8 & 7.3 & 19 & 773 & Antigen avoidance \\
\hline & & $\begin{array}{l}\text { / before and } 6 \\
\text { months after BF }\end{array}$ & & & & \\
\hline IPF & UIP & 18.7 & 9.5 & 29.5 & 797 & Started nintedanib \\
\hline & & $\begin{array}{l}\text { / } 7 \text { months and } \\
1 \text { month before } \\
\text { BF }\end{array}$ & & & & \\
\hline CTD-ILD & UIP & 23 & 8.3 & 14.8 & 536 & $\begin{array}{l}\text { mPSL pulse } \\
\text { started after BF }\end{array}$ \\
\hline & & $\begin{array}{l}16 \text { months and a } \\
\text { week before BF }\end{array}$ & & & & \\
\hline
\end{tabular}


\%FVC, \% forced vital capacity; HRCT, high-resolution computed tomography; Th2, the proportion of Th2 in conventional $C D 4^{+} T$ cells in the BALF; KL-6, Krebs von den Lungen-6; PSL, prednisolone; MPSL, methyl PSL; TAC, tacrolimus; BF, bronchoscopy; IPF, idiopathic pulmonary fibrosis; HP, hypersensitivity pneumonitis; CTD-ILD, connective tissue disease-associated interstitial lung disease; fNSIP, fibrotic nonspecific interstitial pneumonia; UIP, usual interstitial pneumonia; PFT, pulmonary function test

\section{Figures}
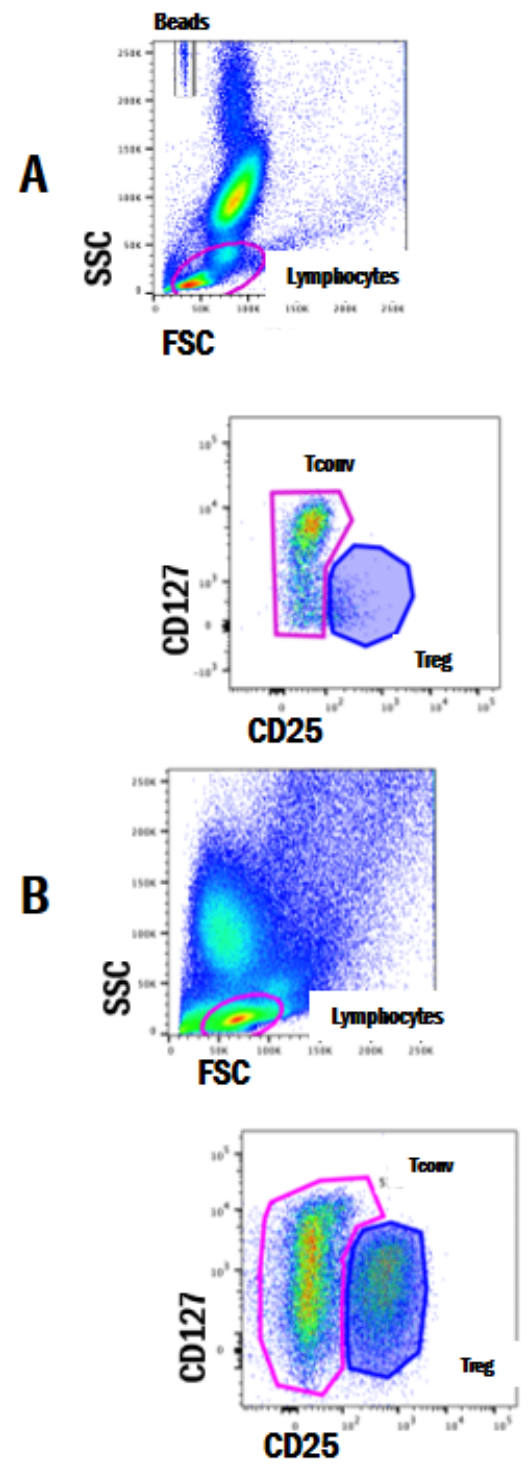
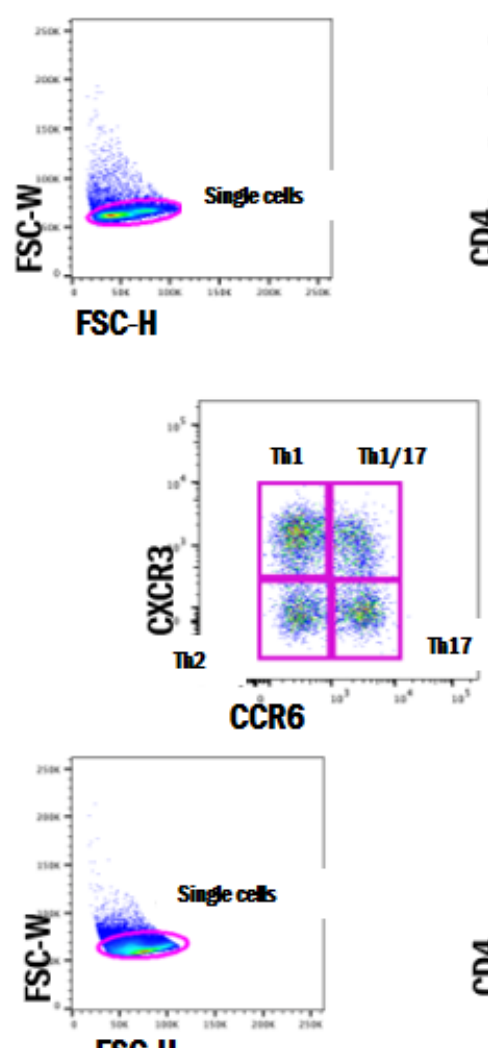

FSC-H

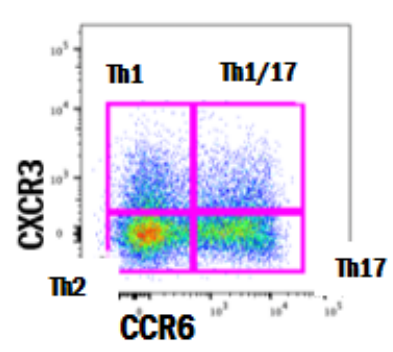

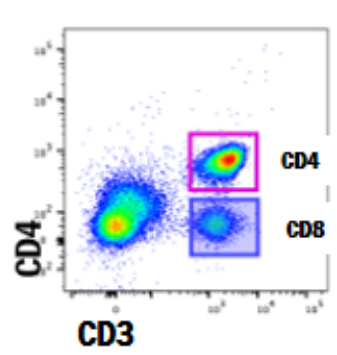
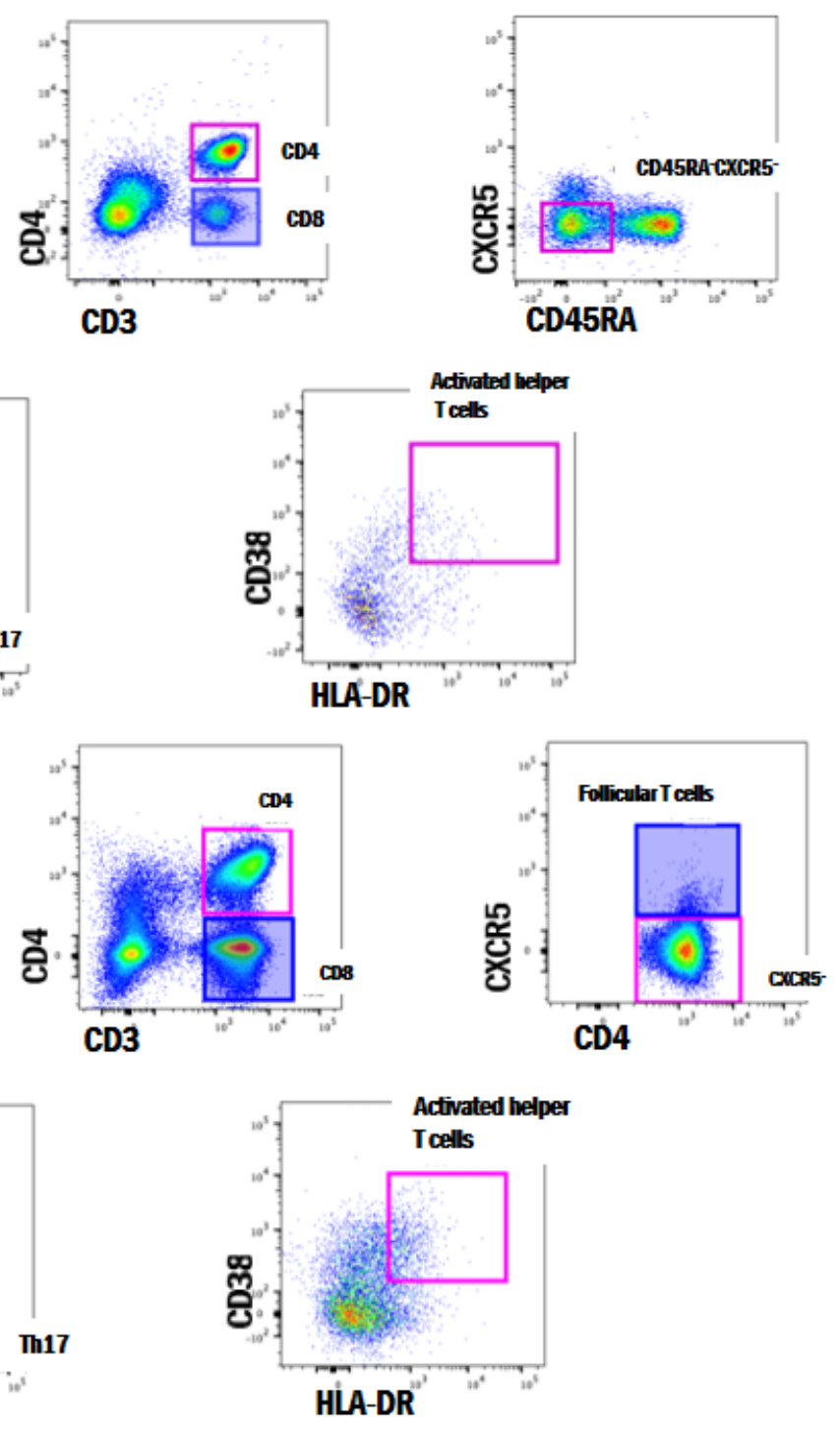

Figure 1

Gating strategy of flow cytometry for identifying helper T cell (Th) subsets 
For analysis of peripheral blood (A), we gated on live lymphocytes using FSC and SSC, followed by exclusion of doublets, then identified $\mathrm{CD} 3^{+} \mathrm{CD} 4^{+} \mathrm{T}$ cells. Among these $\mathrm{CD} 3^{+} \mathrm{CD} 4^{+} \mathrm{T}$ cells, we gated on CD45RA-CXCR5 ${ }^{-}$populations to gate out CD45RA ${ }^{+}$naïve $T$ cells and $C X C R 5^{+}$follicular helper $T$ cells, then gated on CD127 $7^{\text {Low }}$ or CD127 $7^{\text {High }}$ Tconv and to gate out Tregs using CD25 and CD127. CCR6 and CXCR3 for the classification of Th subsets (refer to Table 1). The same gating strategy was applied for analysis of BALF cells (B). In the BALF cells, the activation of each Th subset was identified using HLA-DR and CD38. $A$ and $B$ are examples of mixed connective disease-associated ILD. ILD, interstitial lung disease; BALF, bronchoalveolar lavage fluid; FSC, forward scatter; SSC, side scatter; Tconv, conventional T cells; Tregs, regulatory $T$ cells.

A
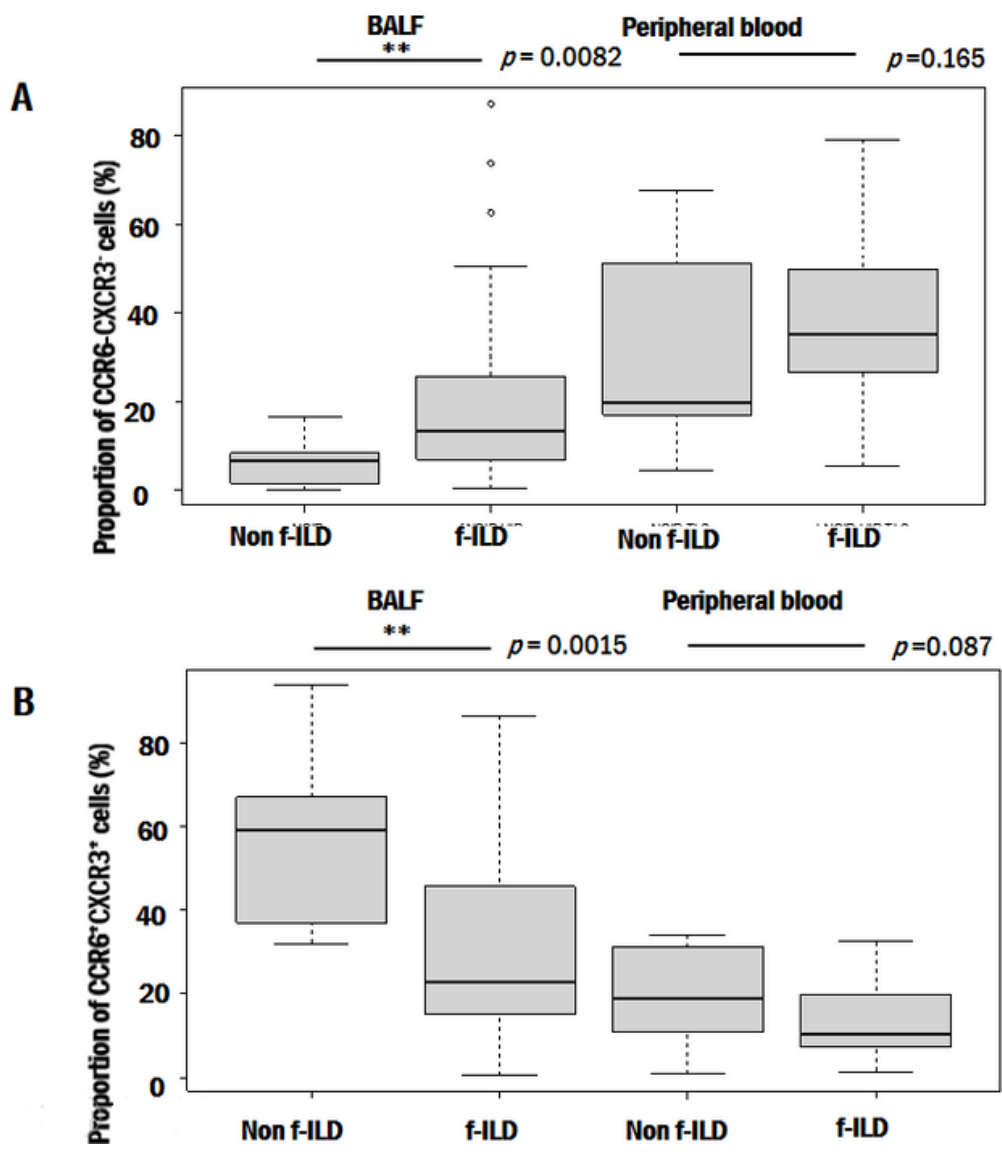

B

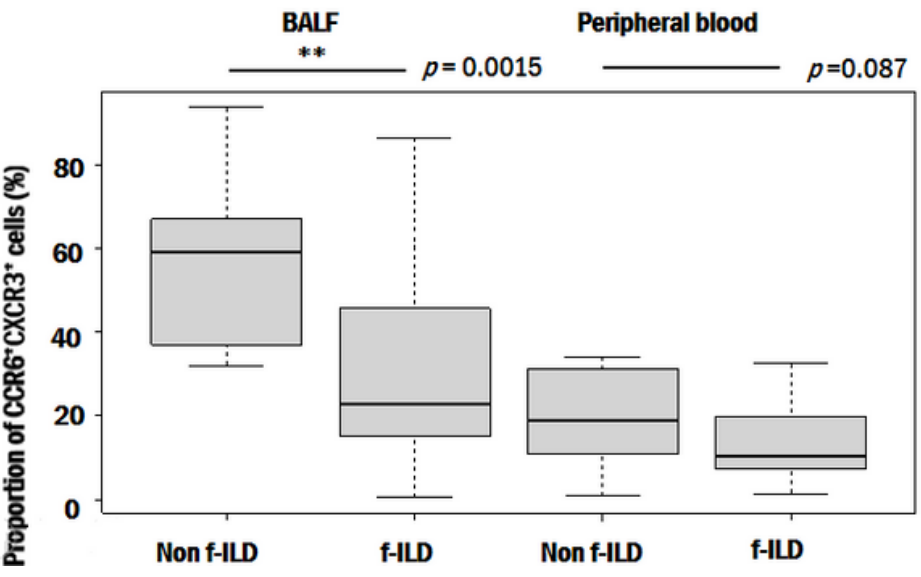

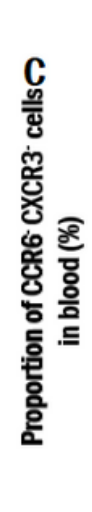
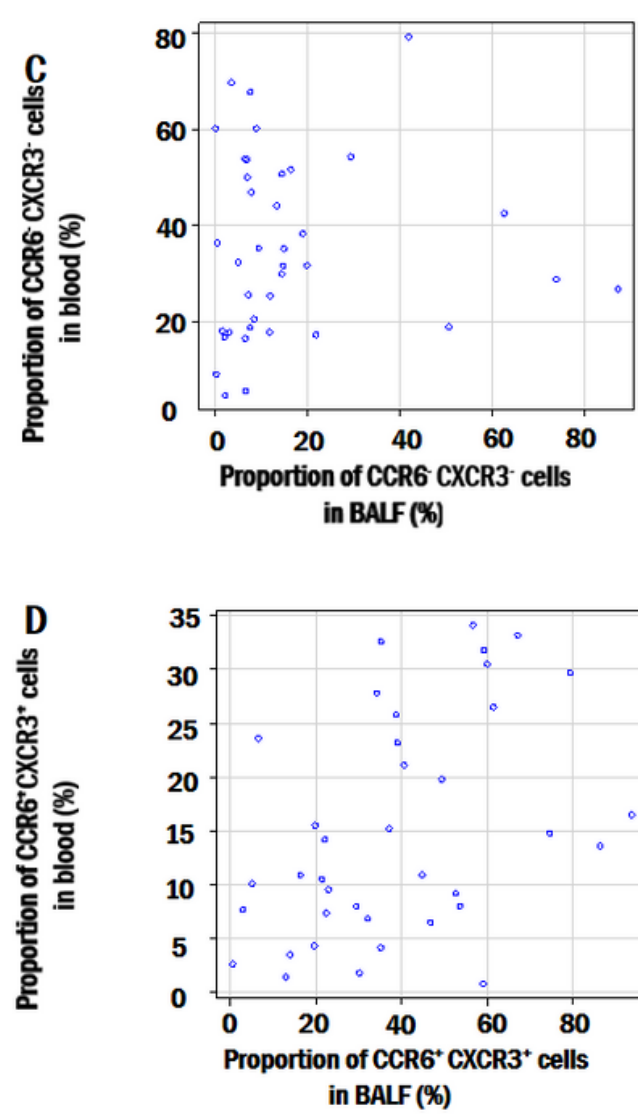

Rs $=0.181$

$p=0.276$

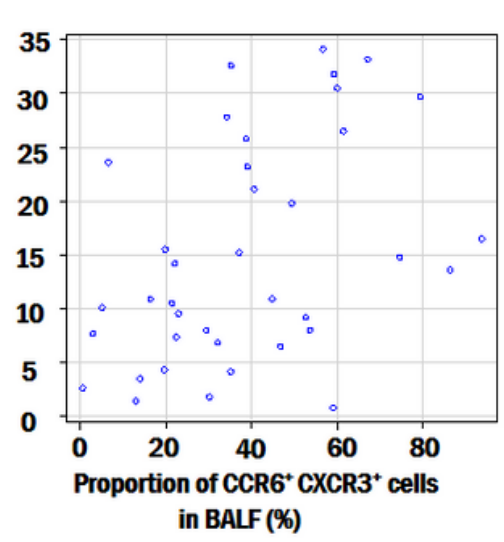

Rs $=0.485$ $p=0.0021$

Figure 2

Increased proportion of the Th2 subset in bronchoalveolar lavage fluid of patients with fibrotic phenotype on chest high-resolution computed tomography

The proportions of CCR6 ${ }^{-} \mathrm{CXCR}^{-}$Th2 (A) and CCR6 ${ }^{+} \mathrm{CXCR}^{+}$Th1/17 (B) in BALF and peripheral blood in patients with non-fibrotic interstitial lung diseases (ILDs) or fibrotic ILDs on chest HRCT. The number of subjects is 13 with non-fibrotic ILDs and 27 with fibrotic ILDs for BALF, and 12 with non-fibrotic ILDs and 26 with fibrotic ILDs for peripheral blood. The correlation between the proportion of CCR6 ${ }^{-}$CXCR3 $^{-}$Th2 (C) and CCR6 $^{+}{ }^{+} \mathrm{CCR}^{+}{ }^{+}$Th1/17 (D) in peripheral blood and in BALF ( $\left.n=38\right)$. f-ILD, fibrotic interstitial lung disease; BALF, bronchoalveolar lavage fluid. 


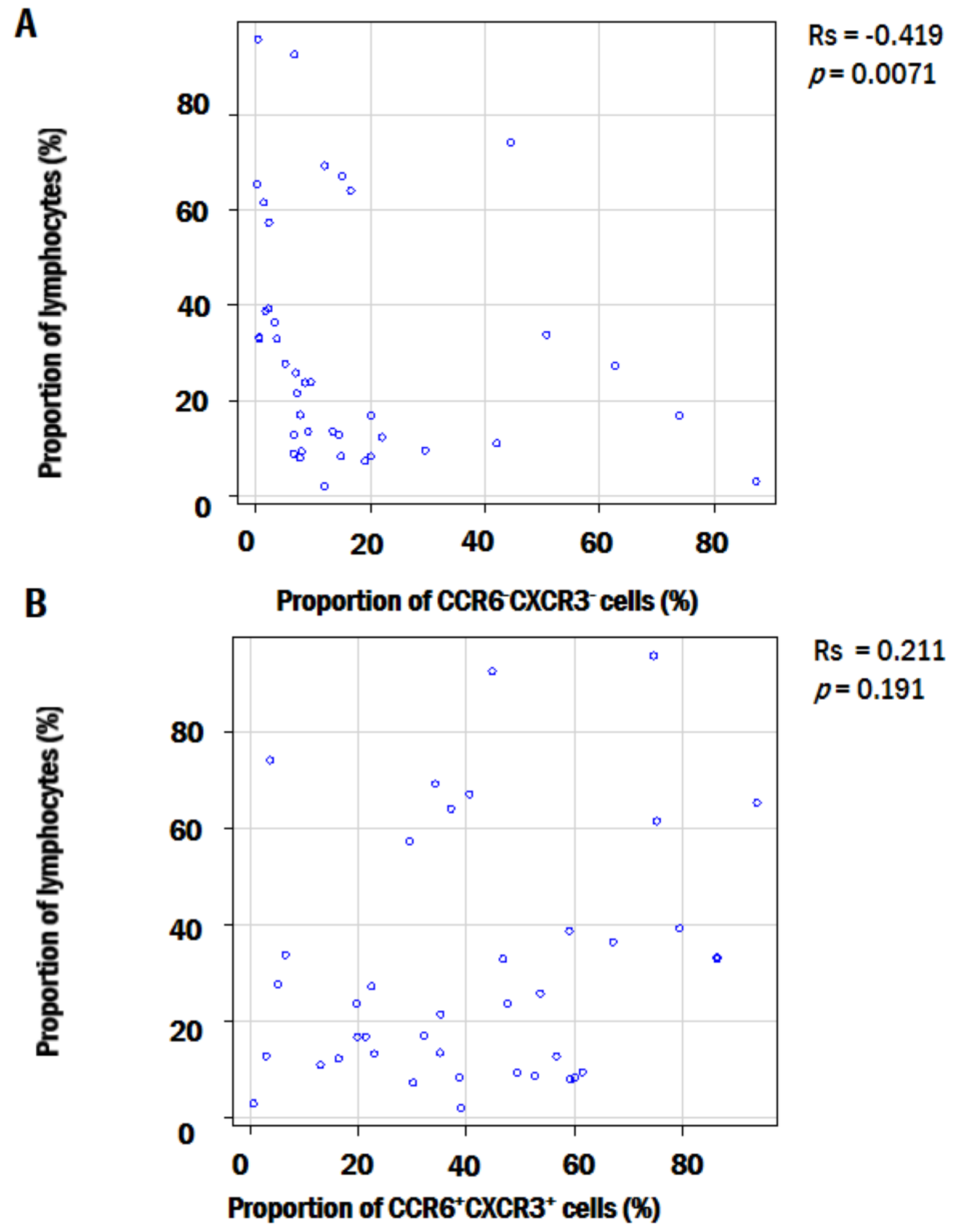

Figure 3

Correlations between the proportion of lymphocytes and CCR6 ${ }^{-}$CXCR3 $^{-}$Th2 $(A)$ or CCR6 ${ }^{+}{ }^{-} X C R 3^{+}$Th $1 / 17$ (B) in BALF of all ILD patients $(n=40)$. BALF, bronchoalveolar lavage fluid. 

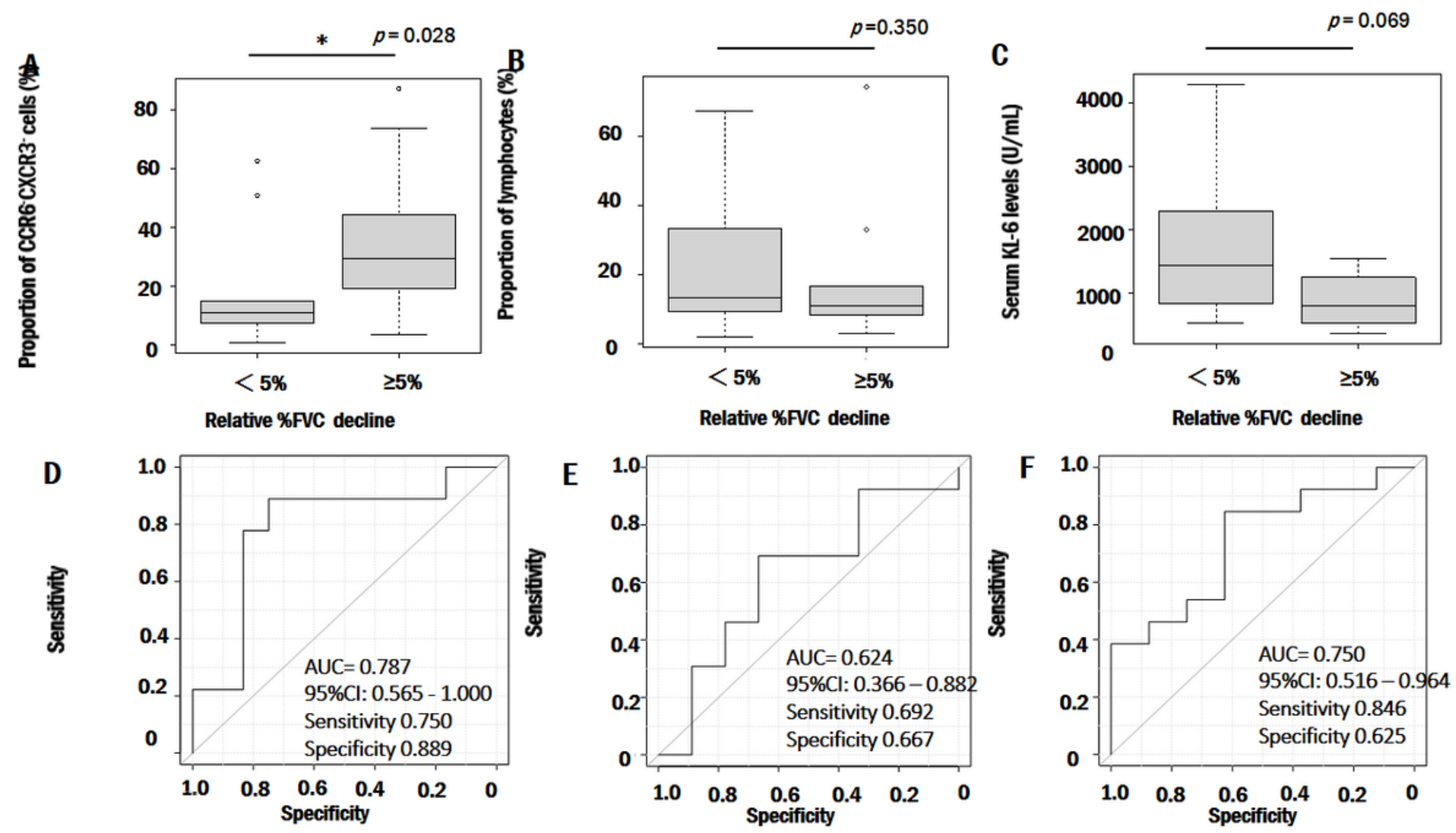

Figure 4

Increased proportion of the Th2 subset in bronchoalveolar lavage fluid in patients with a greater decrease in relative \% forced vital capacity.

The proportion of CCR6 ${ }^{-}$CXCR3- Th2 cells in BALF (A), the proportion of lymphocytes in BALF (B) and serum KL-6 levels $(C)$ in patients with a relative decrease in \%FVC of less than $5 \%(n=12)$ or those with $5 \%$ or more $(n=9)$ over 6 months. Receiver operating characteristic (ROC) analysis of the proportion of Th2 cells in BALF for determining the progressive phenotype of fibrotic ILD patients (D). ROC analysis was also performed for the proportion of lymphocytes in BALF and serum KL-6 levels (E, F).

The details of the relationships among \%FVC decline, chest HRCT findings, diagnosis, and treatment are shown in Table 3. BALF, bronchoalveolar lavage fluid; FVC, forced vital capacity; KL-6, Krebs von den Lungen-6; AUC, area under the curve; $\mathrm{Cl}$, confidence interval.

\section{Supplementary Files}

This is a list of supplementary files associated with this preprint. Click to download.

- Additionalfile1.docx

- Additionalfile2.pptx 